\title{
Navigating the landscape of nonlinear mechanical metamaterials for advanced programmability
}

\author{
Eder Medina, ${ }^{1}$ Patrick E. Farrell, ${ }^{2}$ Katia Bertoldi, ${ }^{1,3,}{ }^{*}$ and Chris H. Rycroft ${ }^{1,4, \dagger}$ \\ ${ }^{1}$ Harvard John A. Paulson School of Engineering and Applied Sciences, Harvard University, Cambridge, Massachusetts 02138, USA \\ ${ }^{2}$ Mathematical Institute, University of Oxford, Oxford OX2 6GG, United Kingdom \\ ${ }^{3}$ Kavli Institute, Harvard University, Cambridge, Massachusetts 02138, USA \\ ${ }^{4}$ Computational Research Division, Lawrence Berkeley Laboratory, Berkeley, California 94720, USA
}

(Received 1 October 2019; revised manuscript received 7 January 2020; accepted 10 January 2020; published 3 February 2020)

\begin{abstract}
We consider a flexible mechanical metamaterial comprising an elastomeric matrix with an embedded square array of circular holes. First, we use the deflated continuation technique of bifurcation analysis to explore its complex energy landscape, characterized by multiple bifurcations from which stable and unstable branches emanate. We then investigate how this landscape can be used to design materials with advanced programmability. We find that the response of the system can be constantly reprogrammed through local manipulation, moving it from one stable branch to another, and that small targeted imperfections can be harnessed to enhance such programmability.
\end{abstract}

DOI: 10.1103/PhysRevB.101.064101

\section{INTRODUCTION}

Mechanical metamaterials-man-made structures with mechanical properties governed by their geometry rather than composition-are receiving increasing interest [1-3] not only because of their rich physics, but also for their unique mechanical properties, including negative Poisson's ratio [4], negative thermal expansion [5], and negative dynamic moduli [6,7]. Intriguingly, it has been also shown that nonlinearities and instabilities can be exploited to further enhance their functionalities [3] and enable the design of systems with switchable properties [8-10] and programmable responses [11-14]. It is no surprise that such flexible systems usually have an intricate energy landscape with a plethora of energy minima $[15,16]$. However, their behavior is typically studied using analyses that capture only a single solution [8-10,13], thus limiting the range of achievable deformation-induced functionalities.

In parallel, continuation and bifurcation analysis techniques, such as simplicial continuation [17], pseudo-arclength continuation [18,19], and branch switching [20], have emerged as powerful tools to obtain insight into the energy landscapes of physical systems. By numerically following the equilibrium equations as a function of a control parameter (e.g., a mechanical or geometrical variable of the problem), these methods enable the computation of additional equilibrium solution branches. While such techniques have been widely used to get a better understanding of numerous physical systems [21-24], their adoption in the design of mechanical metamaterials has been limited $[15,16,25]$.

Here we demonstrate how a novel bifurcation analysis technique in conjunction with the rich energy landscape of flexible mechanical metamaterials can facilitate the realization of systems with advanced programmability. We

\footnotetext{
*bertoldi@ seas.harvard.edu

†chr@seas.harvard.edu
}

consider a flexible and porous metamaterial, and we start by making use of a deflated continuation algorithm to identify solutions, taking advantage of the ability of this algorithm to automatically find disconnected solution branches. Then, we investigate the implications of these numerical analyses on the responses of physical samples. Remarkably, we find that the information encoded in the bifurcation diagrams can be harnessed to realize mechanical metamaterials whose responses can be reconfigured by identifying nearby stable branches and to develop a deeper understanding of the interplay between small targeted imperfections and the evolution of branch connectivity, which can enable advanced programmability.

\section{NUMERICAL METHODS}

We use the open-source finite-element solver FEniCS [26] to numerically investigate the nonlinear response of flexible porous structures made of an elastomeric material whose response is captured using a Neo-Hookean model with a strain energy density function

$$
\psi(\mathbf{F})=\frac{\mu}{2}\left(I_{c}-2\right)-\mu \ln J+\frac{\mu \nu}{1-2 v}(\ln J)^{2},
$$

where $\mu$ is the initial shear modulus, and $v$ is Poisson's ratio. Moreover, $\mathbf{F}=\mathbf{I}+\nabla \mathbf{u}$ is the deformation gradient tensor (with $\mathbf{u}$ being the displacement field to be computed and $\mathbf{I}$ denoting the identity matrix), and $I_{c}=\operatorname{tr} \mathbf{F}^{\mathrm{\top}} \mathbf{F}$ and $J=\operatorname{det} \mathbf{F}$. In all of our analysis, we use plane strain conditions and discretize the models with piecewise quadratic triangular finite elements. This results in a parametrized nonlinear system of equations

$$
\mathbf{R}\left(\mathbf{u}, \varepsilon_{a}\right)=\mathbf{0},
$$

which we solve using the Newton-Raphson method to obtain a displacement field, $\mathbf{u}$, at a given applied strain, $\varepsilon_{a}$. Numerical continuation is employed to trace out the full branch as a function of the applied strain. 


\section{A. Deflated continuation}

Standard finite-element analysis and continuation techniques are only capable of capturing a single solution, reducing the opportunity for programmability. To overcome this limitation, we couple FEniCS with a deflated continuation package DEFCON [27], which enables the identification of additional branches. The key idea of deflation is to modify Eq. (2) to remove known solutions such that the NewtonRaphson method is able to converge to a previously unknown solution. This is analogous to removing a root $x=r$ of a polynomial $p(x)$ by constructing $q(x)=p(x) /(x-r)$. In this work, the deflated problem is constructed as

$$
\mathbf{G}\left(\mathbf{u}, \varepsilon_{a} ; \mathbf{u}^{*}\right)=\left(\frac{1}{\left\|\mathbf{u}-\mathbf{u}^{*}\right\|_{H^{1}}^{2}}+1\right) \mathbf{R}\left(\mathbf{u}, \varepsilon_{a}\right)=\mathbf{0},
$$

where $\|\cdot\|$ denotes the $H^{1}$ norm $\left[\|\cdot\|_{H^{1}}^{2}=\int(\cdot)^{2} d \Omega+\right.$ $\left.\int(\nabla \cdot)^{2} d \Omega\right]$ and $\mathbf{u}^{*}$ is an additional solution. We solve this deflated problem using the same computational techniques as for the undeflated one. Since the deflation operator removes the original solution $\mathbf{u}$ from $\mathbf{R}$ [28], if the Newton-Raphson method converges it will converge to a different solution. We iteratively apply this procedure to obtain as many solutions $\mathbf{u}^{*}$ as possible at a given $\varepsilon_{a}$ through premultiplication on the augmented systems [27].

In this work, we start from $\varepsilon_{a}=0$ and examine the structure in compression, taking small negative steps in $\varepsilon_{a}$. Once a solution has been successfully deflated at a given $\varepsilon_{a}$, additional branches are then further traced out. Let $\varepsilon_{a}^{+}$and $\varepsilon_{a}^{-}$denote the previous and next values of $\varepsilon_{a}$, respectively. After all known branches have been continued, all solutions at $\varepsilon_{a}^{-}$are deflated, and each solution at $\varepsilon_{a}^{+}$is used as an initial guess for the application of Newton-Raphson to the deflated problem. This robustly discovers new solutions introduced at connected bifurcations between $\left(\varepsilon_{a}^{-}, \varepsilon_{a}^{+}\right)$. In addition, deflation can discover disconnected branches, provided the distance between the known and disconnected branch is not too large. Such disconnected branches typically arise from symmetry-breaking perturbations that unfold connected bifurcations; they are important for understanding the behavior of physical systems, but they are generally quite difficult to discover numerically.

\section{B. Stability}

Having discovered a set of solution pairs $\left(\mathbf{u}, \varepsilon_{a}\right)$, we then determine their stability by investigating the propagation of small-amplitude oscillatory modes

$$
\mathbf{W}\left(\mathbf{x}_{0}, t\right)=\mathbf{w}\left(\mathbf{x}_{0}\right) e^{-i \omega t}
$$

superimposed upon a given state of finite deformation defined by $\mathbf{u}, \omega$ being the oscillation frequency and $\mathbf{x}_{0}$ denoting the position of a material point in the undeformed configuration. More specifically, we consider the incremental version of the equations of motion in the reference undeformed configuration [29],

$$
\operatorname{Div} \dot{\mathbf{S}}=\rho \frac{D^{2} \mathbf{W}}{D t^{2}}
$$

where $\rho$ is the material density, Div denotes the div operator in the reference configuration, and $\frac{D}{D t}$ is the Lagrangian time derivative. Moreover, $\dot{\mathbf{S}}$ is the increment of total first PiolaKirchhoff stress, which can be written as

$$
\dot{\mathbf{S}}=\mathbb{L}: \dot{\mathbf{F}}
$$

with

and

$$
\mathbb{L}_{i j k l}=\frac{\partial^{2} \psi}{\partial F_{i j} \partial F_{k l}}
$$

$$
\dot{\mathbf{F}}=\frac{\partial \mathbf{W}}{\partial \mathbf{x}_{0}},
$$

where $\psi$ is the strain energy density and $\mathbf{F}$ is the deformation gradient. Substitution of Eq. (4) into Eqs. (6) and (5) yields

$$
\dot{\mathbf{S}}\left(\mathbf{x}_{0}, t\right)=\mathbb{L}: \frac{d \mathbf{w}\left(\mathbf{x}_{0}\right)}{d \mathbf{x}_{0}} e^{-i \omega t}
$$

and

$$
\operatorname{Div}\left(\mathbb{L}: \frac{d \mathbf{w}\left(\mathbf{x}_{0}\right)}{d \mathbf{x}_{0}}\right)+\rho \omega^{2} \mathbf{w}=\mathbf{0},
$$

which when discretized using the finite-element approach becomes

$$
\left[\mathbf{K}(\mathbf{u})+\omega^{2} \mathbf{M}\right] \mathbf{w}=\mathbf{0},
$$

where $\mathbf{K}$ is the tangent stiffness matrix and $\mathbf{M}$ is the standard mass matrix. We introduce a normalization parameter,

$$
\lambda=\frac{\rho L^{2}}{\mu} \omega^{2}
$$

with $L$ being the length of the structure. A positive $\lambda$ corresponds to a vibration of finite amplitude, and a negative $\lambda$ identifies a perturbation growing exponentially with time. Therefore, the deformed configuration defined by $\mathbf{u}$ is stable for $\lambda_{\min } \equiv \min (\lambda)>0$ and unstable for $\lambda_{\min }<0$.

\section{RESULTS}

We focus on structures consisting of an elastomeric matrix with an embedded square array of circular holes, a relatively simple architecture that is capable of giving rise to a rich complex energy landscape $[8,16,25,30,31]$. The holes have radius $r$ and center-to-center distance $a$, chosen so that the initial porosity is $\psi_{0}=\pi r^{2} / a^{2}=0.6$. Two different finitesize structures are considered: one with a $2 \times 2$ array of holes and another with a $3 \times 3$ array of holes. All structures have the two vertical edges flanked by a column of semicircles and the horizontal ones ending with a strip of solid material of width $a$. They are made of an elastomeric material, whose response is captured by a quasi-incompressible neo-Hookean model with initial shear modulus $\mu=49 \mathrm{kPa}$ and Poisson's ratio $v=0.48$.

\section{A. Bifurcation analysis}

In Figs. 1(a) and 1(b) we report the evolution of the normalized nominal stress, $\tilde{S}=S_{22} / \mu$, as a function of the applied strain, $\varepsilon_{a}$, for the $2 \times 2$ and $3 \times 3$ structures, respectively. As expected, we find that the response of both systems is characterized by multiple branches, some of which are stable and 


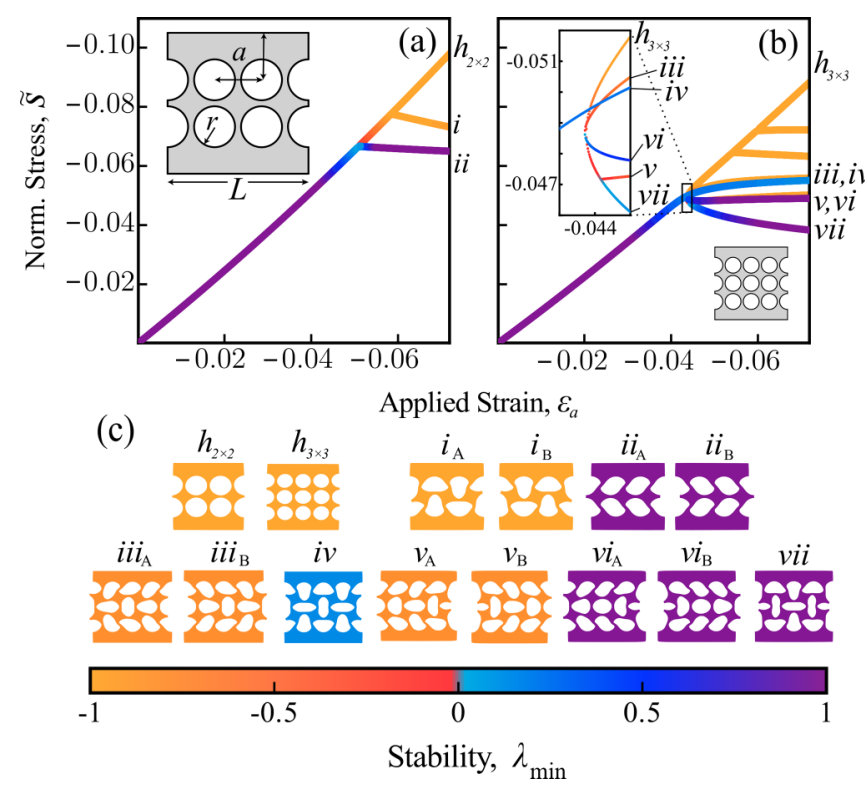

FIG. 1. (a), (b) Computed bifurcation diagrams for structures with an array of (a) $2 \times 2$ and (b) $3 \times 3$ holes. The color of the branches corresponds to the minimal eigenvalue $\lambda_{\min }$, a measure of stability. The inset gray diagrams show the undeformed structures. The inset in panel (b) shows a zoomed-in region near the bifurcation. (c) Solutions corresponding to the labeled branches $h_{2 \times 2}, h_{3 \times 3}$, and $i-v i i$ at $\varepsilon_{a}=-0.07$. Collections of branches are labeled based on having an identical mechanical response, and they are sublabeled with capital letters to differentiate between left and right symmetries.

some unstable. Moreover, as previously observed for porous structures of small size $[15,32]$, our results also indicate that boundary effects present in finite-sized structures play an important role, as the bifurcation diagrams of the $2 \times 2$ and $3 \times 3$ structures are significantly different. For the considered range of applied strain, the response of the $2 \times 2$ structure is characterized by two pitchfork bifurcations. The first one at $\varepsilon_{a} \approx-0.051$ gives rise to a stable sheared post-buckled solution with all holes sheared either leftwards or rightwards, whereas from the second one at $\varepsilon_{a} \approx-0.058$ an unstable branch emanates, corresponding to a polarized configuration in which the holes alternately take horizontally and vertically dominant shapes.

The bifurcation diagram of the $3 \times 3$ structure is more intricate and, in addition to multiple unstable branches stemming from bifurcations off the main branch, contains a bifurcation point at $\varepsilon_{a} \approx-0.043$ from which three stable branches appear to emanate. Closer inspection shows that this bifurcation does not occur at a single point [Fig. 1(b), inset], but consists of a series of disconnected transcritical bifurcations similar to those previously observed in holey columns [15] (for transcritical branch classification analysis, see Figs. S4S5 of the Supplemental Material [33]). Moreover, we find that for the $3 \times 3$ structure, both the sheared and polarized modes are stable. However, while the two symmetric sheared solutions share the same stress-strain curve [i.e., branches $v i_{\mathrm{A}}$ and $v i_{\mathrm{B}}$ in Fig. 1(b)], the two supported polarized solutions are associated with two distinct branches: the one where the central hole is vertically oriented with branch $i v$ (which is connected to the main branch, but is only marginally stable),
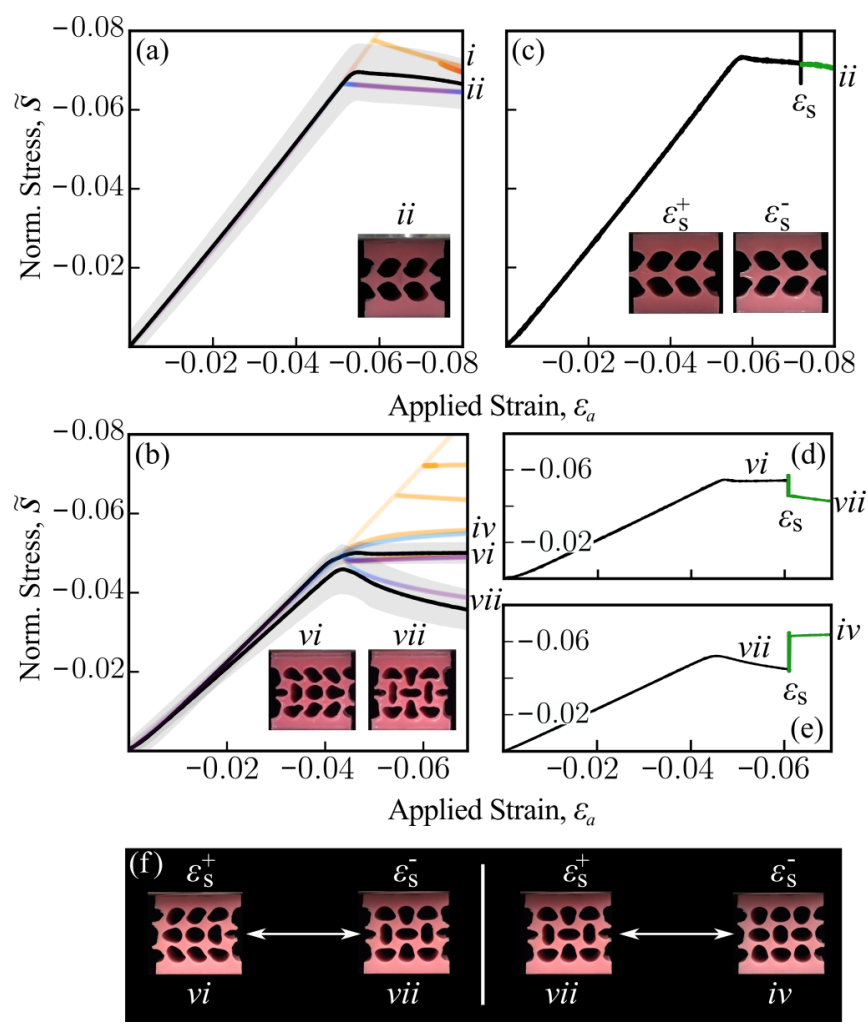

FIG. 2. (a) Experimentally measured stress-strain curve for the $2 \times 2$ structure, overlaid on the numerical bifurcation diagram from Fig. 1(a) (colored lines). The solid black line shows the average response for four samples, and the gray envelope shows the standard deviation. The inset shows a deformed sample at $\varepsilon_{a}=-0.07$, in the sheared $i i_{\mathrm{A}}$ mode. (b) Experimentally measured stress-strain curves for the $3 \times 3$ structure. In 10 samples tested, 7 follow branch $v i$ and 3 follow branch vii; the averages and standard deviations for each set are plotted separately. The insets show deformed samples in both deformation modes at $\varepsilon_{a}=-0.07$. (c) Stress-strain curve for a single $2 \times 2$ structure that is manually switched from branch $i i_{\mathrm{A}}$ to $i i_{\mathrm{B}}$ at $\varepsilon_{S}=-0.072$. The curve after the switch is shown in green. Insets show the structure before $\left(\varepsilon_{S}^{+}\right)$and after $\left(\varepsilon_{S}^{-}\right)$the switch. $(\mathrm{d}, \mathrm{e})$ Stress-strain curves for two differing $3 \times 3$ structures that are manually switched from one branch to another at $\varepsilon_{S}=-0.06$. (f) Images of the $3 \times 3$ structures before and after the switches.

and the one where the central hole is horizontally oriented with branch vii. Having identified multiple solution branches under uniaxial compression, in the remainder of this paper we investigate how this energy landscape can be exploited.

\section{B. Experiments}

Next, we focus on the implications of the bifurcation diagrams on the response of physical samples fabricated using a molding approach out of a silicone rubber (Elite Double 8, Zhermack). We fabricate several $2 \times 2$ and $3 \times 3$ structures using different molds and then characterize their response under uniaxial compression. As shown in Fig. 2(a), we find that for all $2 \times 2$ samples, the sheared mode emerges upon compression beyond the buckling point. Differently, none of $3 \times 3$ structures deform according to the fully connected branch (i.e., branch $i v$ ), which a standard finite-element simulation 


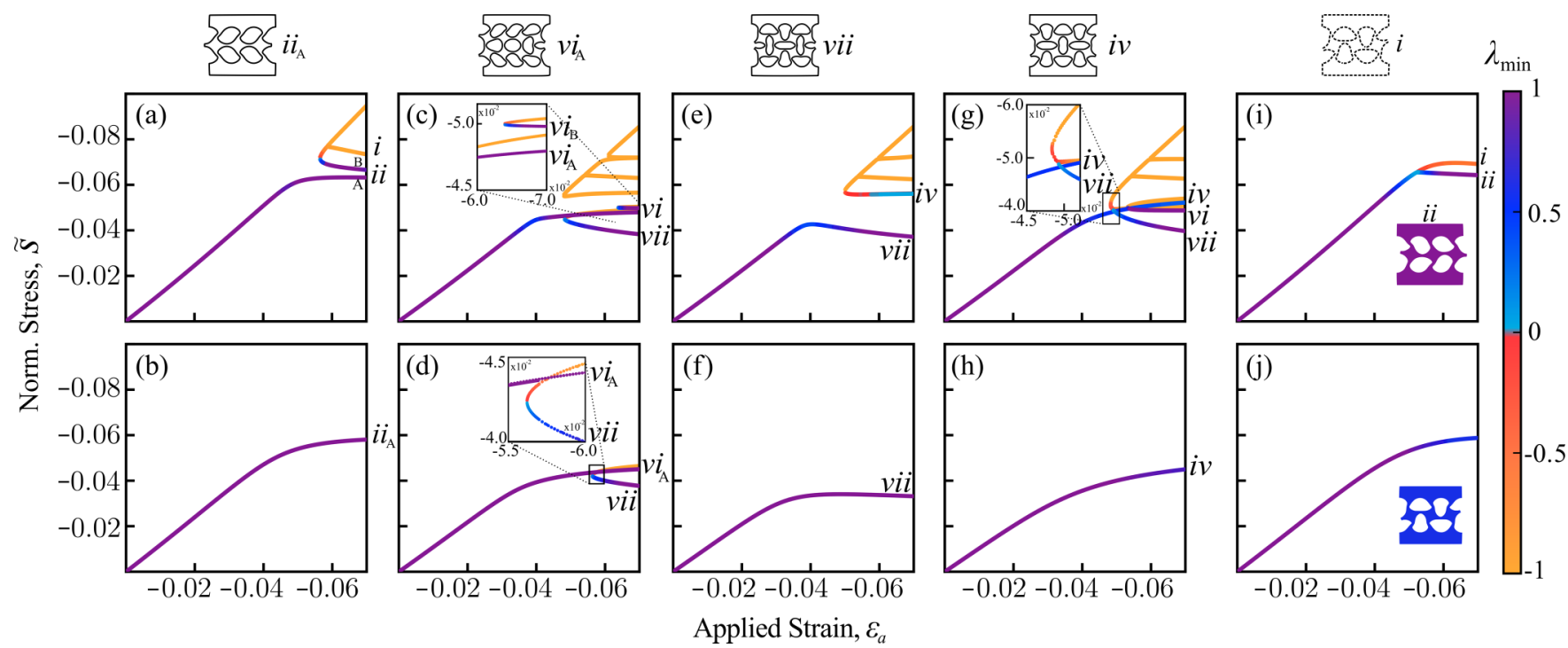

FIG. 3. Bifurcation diagrams for the perturbed structures using imperfection magnitudes of $\delta=5 \times 10^{-4}$ (top row) and $\delta=2 \times 10^{-3}$ (bottom row). The branches are colored according to the stability measure $\lambda_{\min }$. The five columns correspond to perturbations based on the structures from branches $i_{\mathrm{A}}, i v_{\mathrm{C}}, v, i i i, i$ in Fig. 1, which are plotted at the top of each column; the first four columns use perturbations based on stable branches, and the last column uses a perturbation based on an unstable branch. The images in panels (i) and (j) show the deformed structures at the end of two branches at $\varepsilon_{a}=-0.07$.

would follow (Fig. S2). Instead, they all deform according to either branches $v i_{\mathrm{A}, \mathrm{B}}$ or vii [Fig. 2(b)]. We attribute this to immeasurable small imperfections introduced during fabrication that cause changes to the symmetry group of the system and, ultimately, to the branch connectivity [34]. However, it is important to note that, though disconnected, other stable branches should still exist under such small imperfections. To probe their existence, we compress a sample up to a switching strain $\varepsilon_{S}$ past the critical buckling point, locally manipulate its free boundary to move it onto another branch, and then further compress it. As shown in Fig. 2(c), we find that the $2 \times 2$ samples can always be moved to the other symmetric sheared mode. Such reconfiguration only leads to a change in geometry, since the two branches are characterized by an identical stress-strain curve. Differently, for the $3 \times 3$ structures we find that not all initially predicted stable branches can be accessed directly nor can they be accessed by locally manipulating the samples at the chosen $\varepsilon_{s}$. Specifically, samples that deform following branch vii can only be reconfigured to the polarized mode associated to branch $i v$ [Fig. 2(d)], whereas those that follow branches $v i_{\mathrm{A}, \mathrm{B}}$ can only be reconfigured to branch vii [Fig. 2(e)]. Since these three branches are characterized by different stress-strain curves, branch switches are also accompanied by a change in stiffness and load-bearing capacity. Note that, since the system is purely elastic, such branch switches are reversible and repeatable (see Movie S2 in the Supplemental Material [33]), suggesting that the response of our mechanical metamaterial can be easily reprogrammed at any time by taking it from one stable branch to another.

\section{Imperfection analysis}

The results of Fig. 2 indicate that the information encoded in the bifurcation diagram can be used to program the response of the metamaterials on the fly. However, they also show that not all stable branches can be accessed, limiting the available range of programmability. To gain a better understanding of the reasons behind this limitation, we proceed by constructing the bifurcation diagrams for structures with initial mode-derived perturbations. Toward this end, we apply to the undeformed structures a perturbation field $\mathbf{u}_{\text {pert }}$ defined as

$$
\mathbf{u}_{\text {pert }}=\delta \frac{\mathbf{u}_{\text {branch }}-\mathbf{u}_{h_{i \times i}}}{\left\|\mathbf{u}_{\text {branch }}-\mathbf{u}_{h_{i \times i}}\right\|_{L^{2}}}
$$

where $\delta$ is the imperfection magnitude, and $\mathbf{u}_{\text {branch }}$ and $\mathbf{u}_{h_{\text {ixi }}}$ (with $i=2,3$ ) are the displacement fields at $\varepsilon_{a}=-0.07$ associated with the branch of interest and the $h_{i \times i}$ branch (Fig. 1), respectively. In Fig. 3 we present the bifurcation diagrams obtained for different mode-derived imperfections for $\delta=5 \times 10^{-4}$ (top row) and $\delta=2 \times 10^{-3}$ (bottom row). To understand our experimental results better, we focus first on Figs. 3(a), 3(c) and 3(e), where we consider small perturbations with the form of the modes that emerge during our experiments (i.e., the modes associated with branches $i i_{\mathrm{A}}, v i_{\mathrm{A}}$, and vii). For all three cases, we find that the complex energy landscape of our structures is preserved, though modified. More specifically, for the $2 \times 2$ structure the $i_{\mathrm{A}}$-perturbation (i.e., an imperfection based on the $i i_{\mathrm{A}}$ branch) separates the sheared modes, but preserves both of them [Fig. 3(a)], allowing for the system to be easily reconfigured from one to the other [Fig. 2(c)]. Differently, for the $3 \times 3$ structure the considered imperfections not only alter the connectivity of the branches, but also suppress some of them. In particular, the $v i_{\mathrm{A}}$-perturbation eliminates the response associated with branch $i v$ [Fig. 3(c)], while the vii-perturbation suppresses that associated with branch $v i[$ Fig. 3(e)]. Note that these results support the response observed in our experiments [compare Figs. 2(d) and 2(e)] and suggest that the immeasurably small imperfections introduced during fabrication have suppressed 


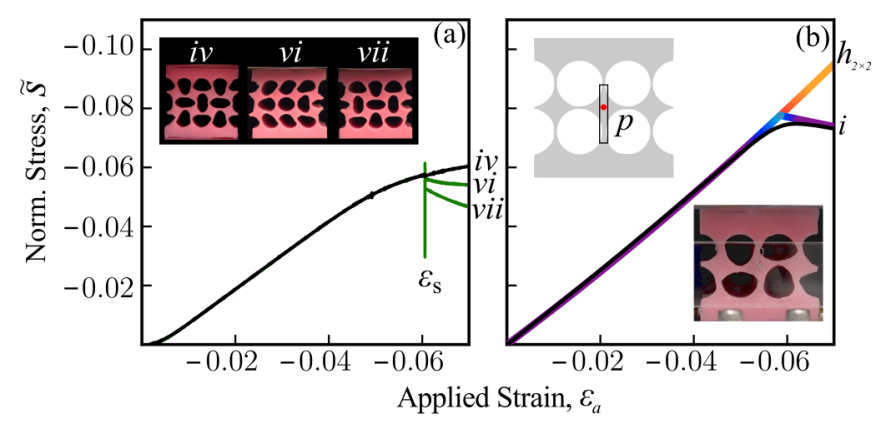

FIG. 4. (a) Experimental stress-strain curve (black) for the $3 \times$ 3 structure with an $i v$ perturbation. For any applied $\varepsilon_{a}$ past the bifurcation point, all three previously predicted stable branches are realizable via manual switching. The stress-strain curves after switching are shown in green and include the noise introduced during the switching process (green vertical line). The inset images show the sample in the three different stable branches. (b) Bifurcation diagram for a $2 \times 2$ structure with its central point (red dot) constrained to move vertically. Colored lines show the simulation results using the same color scheme as in Fig. 1. The solid line shows the experimental stress-strain curve, with the experimental snapshot showing the resulting polarized mode. All experimental images are captured at $\varepsilon_{a}=-0.07$.

branches, which, therefore, cannot be attained through manual reconfiguration at the specified switching strain $\varepsilon_{S}$. Finally, we find that by slightly increasing $\delta$ to $2 \times 10^{-3}$ (which changes the hole areas by less than $1 \%$ ), the intricate bifurcating structure completely vanishes and a single smoothly varying curve emerges. As previously observed [35,36], these results confirm that the introduction of sizable buckling modederived imperfections causes the metamaterial to robustly follow the targeted deformation path. However, by suppressing all other branches, such imperfections limit the response of the system to only one specific mode and prevent any type of switchability.

Next, we analyze the effects of a perturbation based on branch $i v$, a stable branch that is never followed in our experimental tests. As shown in Fig. 3(g), we find that for this perturbation, branch $i v$ is stable and fully connected, all other stable branches still exist, and the transcritical bifurcations remain, though their location is shifted above the main connected branch [Fig. 3(g), inset]. As such, we expect that a sample with an $i v$-perturbation will follow branch $i v$ upon loading and will offer the largest set of programmable properties, since it can be switched to both branches $v i$ and vii. This is confirmed by the experimental results in Fig. 4(a) for a sample realized using a mold designed to introduce an $i v$-perturbation with $\delta=5 \times 10^{-4}$. In all our tests, the sample initially deforms following branch $i v$, and at strain $\varepsilon_{S}=-0.06$ it can be locally manipulated to switch to either branch $v i$ or vii, thus supporting three responses that can be continuously accessed.

\section{Branch stabilization}

Lastly, we investigate the response of the $2 \times 2$ structure with an imperfection based on the unstable branch $i$ [Figs. 3(i) and $3(\mathrm{j})]$. In this case, we find that for $\delta=5 \times 10^{-4}$ branch $i$ remains unstable and that the $h_{2 \times 2}$ branch vanishes, hence the system can only deform following the stable sheared mode. To realize the desired polarized mode, the magnitude of the imperfection has to be increased to $\delta=2 \times 10^{-3}$, but this changes the architecture of the structure and, therefore, significantly affects its stress-strain response, which loses the sharp transition associated with the instability. As we are limited in our ability to achieve the desired response of the unstable modes through targeted imperfection and manual reconfiguration, we therefore turn to imposing additional constraints on our original system and explore their effects on the bifurcation diagram. To suppress the stable sheared mode, we place a pin in the center point of the sample [Fig. 4(b), inset, top left] and guide it along a vertical trajectory. In Fig. 4(b) we show both the bifurcation diagram for the constrained structure as well as the experimentally measured stress-strain curve. Two key features emerge. First, the sheared branch is suppressed, and second, the polarized branch is stabilized. Moreover, the constraint does not affect the mechanical properties of branch $i$, preserving key features such as negative stiffness and a sharp buckling transition.

\section{DISCUSSION AND CONCLUSION}

In summary, we have shown that the deflated continuation technique can be used to explore the complex energy landscapes of flexible mechanical metamaterials and provide insight into the design of multistable systems with advanced functionalities. More specifically, we used the numerically obtained bifurcation diagrams to design a metamaterial whose mechanical properties can be switched on the fly by taking it from one stable branch to another. In addition, we identified the role played by imperfections and mechanical constraints on the bifurcation diagrams and, ultimately, on the response of the metamaterials.

To guide the design of programmable mechanical materials, in this study we considered a two-dimensional structure of relatively small size and simple geometry. We manually perturbed and physically constrained it to move it between different branches. While this enabled us to demonstrate the power of our approach, real-world applications require systems of arbitrary size and shape and the ability to reprogram the response using remote stimuli. Toward this end, it is important to note that the proposed numerical approach can be readily extended to systems of arbitrary size and shape, which will likely be characterized by markedly different bifurcation diagrams. Moreover, depending on the characteristic size of the structures and on their stiffness, different and remote types of stimuli can be used to move the structure between different branches, including magnetic field [37], heat [38,39], and swelling [40]. As such, we believe that our strategy enables the design of a new class of reprogrammable mechanical metamaterials.

\section{ACKNOWLEDGMENTS}

K.B. and C.H.R. acknowledge support from the National Science Foundation under Grant No. DMR-1420570. P.E.F. acknowledges support from the Engineering and Physical Sciences Research Council under Grant No. EP/R029423/1. 
[1] M. Kadic, T. Bückmann, R. Schittny, and M. Wegener, Rep. Prog. Phys. 76, 126501 (2013).

[2] J. Christensen, M. Kadic, O. Kraft, and M. Wegener, MRS Commun. 5, 453 (2015).

[3] K. Bertoldi, V. Vitelli, J. Christensen, and M. van Hecke, Nat. Rev. Mater. 2, 17066 (2017).

[4] G. N. Greaves, A. L. Greer, R. S. Lakes, and T. Rouxel, Nat. Mater. 10, 823 (2011).

[5] Q. Wang, J. A. Jackson, Q. Ge, J. B. Hopkins, C. M. Spadaccini, and N. X. Fang, Phys. Rev. Lett. 117, 175901 (2016).

[6] N. Fang, D. Xi, J. Xu, M. Ambati, W. Srituravanich, C. Sun, and X. Zhang, Nat. Mater. 5, 452 (2006).

[7] S. A. Cummer, J. Christensen, and A. Alù, Nat. Rev. Mater. 1, 16001 (2016).

[8] K. Bertoldi, P. M. Reis, S. Willshaw, and T. Mullin, Adv. Mater. 22, 361 (2010).

[9] S. Babaee, J. Shim, J. C. Weaver, E. R. Chen, N. Patel, and K. Bertoldi, Adv. Mater. 25, 5044 (2013).

[10] P. Wang, F. Casadei, S. Shan, J. C. Weaver, and K. Bertoldi, Phys. Rev. Lett. 113, 014301 (2014).

[11] B. Florijn, C. Coulais, and M. van Hecke, Phys. Rev. Lett. 113, 175503 (2014).

[12] B. Haghpanah, L. Salari-Sharif, P. Pourrajab, J. Hopkins, and L. Valdevit, Adv. Mater. 28, 8065 (2016).

[13] C. Coulais, A. Sabbadini, F. Vink, and M. van Hecke, Nature (London) 561, 512 (2018).

[14] R. M. Neville, R. M. J. Groh, A. Pirrera, and M. Schenk, Phys. Rev. Lett. 120, 254101 (2018).

[15] D. Pihler-Puzović, A. L. Hazel, and T. Mullin, Soft Matter 12, 7112 (2016).

[16] N. Kidambi, R. L. Harne, and K.-W. Wang, Phys. Rev. E 98, 043001 (2018).

[17] E. L. Allgower and K. Georg, Numerical Continuation Methods: An Introduction, Springer Series in Computational Mathematics (Springer-Verlag, Berlin, 1990).

[18] H. B. Keller, in Applications of Bifurcation Theory, edited by P. Rabinowitz (Academic, New York, 1977), pp. 359-384.

[19] T. F. C. Chan and H. B. Keller, SIAM J. Sci. Stat. Comput. 3, 173 (1982).

[20] E. J. Doedel, Congr. Numer. 30, 265 (1981).

[21] J. Sieber, SIAM J. Appl. Dyn. Syst. 1, 248 (2002).
[22] D. M. Ambrose and J. Wilkening, Commun. Appl. Math. Comput. Sci. 4, 177 (2009).

[23] J. Wilkening, Phys. Rev. Lett. 107, 184501 (2011).

[24] C. H. Rycroft and J. Wilkening, J. Comput. Phys. 255, 612 (2013).

[25] C. G. Johnson, U. Jain, A. L. Hazel, D. Pihler-Puzovic, and T. Mullin, Proc. R. Soc. A 473, 20170477 (2017).

[26] A. Logg, K. A. Mardal, G. N. Wells et al., Automated Solution of Differential Equations by the Finite Element Method (Springer, Berlin, Heidelberg, 2012).

[27] P. E. Farrell, C. H. L. Beentjes, and A. Birkisson, arXiv:1603/00809.

[28] P. E. Farrell, A. Birkisson, and S. W. Funke, SIAM J. Sci. Comput. 37, A2026 (2015).

[29] R. Ogden, Non-linear Elastic Deformations, Dover Civil and Mechanical Engineering (Dover, Mineola, New York, 1997).

[30] T. Mullin, S. Deschanel, K. Bertoldi, and M. C. Boyce, Phys. Rev. Lett. 99, 084301 (2007).

[31] Y. Zhang, E. A. Matsumoto, A. Peter, P.-C. Lin, R. D. Kamien, and S. Yang, Nano Lett. 8, 1192 (2008).

[32] C. Coulais, C. Kettenis, and M. van Hecke, Nat. Phys. 14, 40 (2017).

[33] See Supplemental Material at http://link.aps.org/supplemental/ 10.1103/PhysRevB.101.064101 for additional numerical results, details on experiments and fabrication, plus information on data and code availability.

[34] M. Golubitsky, I. Stewart, and D. Schaeffer, Singularities and Groups in Bifurcation Theory: Volume II, Applied Mathematical Sciences Vol. 69 (Springer, New York, 1988).

[35] G. Wu, Y. Cho, I. Choi, D. Ge, J. Li, H. N. Han, T. Lubensky, and S. Yang, Adv. Mater. 27, 2747 (2015).

[36] S. Janbaz, F. S. L. Bobbert, M. J. Mirzaali, and A. A. Zadpoor, Mater. Horiz. 6, 1138 (2019).

[37] Y. Kim, H. Yuk, R. Zhao, S. A. Chester, and X. Zhao, Nature (London) 558, 274 (2018).

[38] T. G. Leong, C. L. Randall, B. R. Benson, N. Bassik, G. M. Stern, and D. H. Gracias, Proc. Natl. Acad. Sci. USA 106, 703 (2009).

[39] Y. Liu, J. K. Boyles, J. Genzer, and M. D. Dickey, Soft Matter 8, 1764 (2012).

[40] J. Kim, J. A. Hanna, M. Byun, C. D. Santangelo, and R. C. Hayward, Science 335, 1201 (2012). 